\title{
Today, you walk! - When Physical Fitness Influences Trip Planning
}

\author{
Stefan Diewald ${ }^{1}$, Andreas Möller ${ }^{1}$, Luis Roalter ${ }^{1}$, Matthias Kranz ${ }^{2}$ \\ Distributed Multimodal Information Processing Group, Technische Universität München ${ }^{1}$ \\ Lehrstuhl für Informatik mit Schwerpunkt Eingebettete Systeme, Universität Passau ${ }^{2}$
}

\begin{abstract}
Technology-based self-tracking of physical activity is a new trend. However, monitoring one's own behavior does not automatically motivate one to increase physical exercising. For that reason, we propose a concept that uses the tracked data for providing fitness routes in everyday trip planning, which are tailored to the users' needs, personal preferences, and fitness levels. We argue that utilizing the monitored data for directed exercising during daily routines can add value to the quantified-self movement.
\end{abstract}

\section{Introduction and Background}

Do you know what you did today, one year ago? You don't? - Some people do. And they can even tell you where they were, for how long, and with whom. The so-called Quantified-Selfers (Choe et al. 2014) track many kinds of data about themselves. With the support of self-tracking technologies, they diligently record their lives and collect large amounts of data that are often stored and processed by cloud systems. Besides those extreme users that track everything in their lives, there is also a broader trend of carrying fitness trackers around. Those trackers are either realized as small gadgets, such as the Fitbit One, Nike+ FuelBand, or Jawbone UP ${ }^{l}$, or apps for smartphones, such as Moves or Runtastic Pedometer ${ }^{2}$. In contrast to sports trackers that are normally only carried and used during exercising (Möller et al. 2012), the fitness trackers are carried the whole day and continually measure steps taken, stairs climbed, overall physical activity intensity, distance traveled, hours slept, and quality of sleep. After uploading the data into the cloud, modern dashboards present activity and sleep graphs as well as abstract numbers of summed up steps and floors for each day, week, month, or overall.

Although having those numbers in black and white allows analyzing shortcomings (e.g., missing the 10,000 steps a day to meet minimum physical activity guidelines, cf. Le-Masurier

1 https://www.fitbit.com/one, http://www.nike.com/us/en_us/c/nikeplus-fuelband, https://jawbone.com/up

2 http://www.moves-app.com/, https://www.runtastic.com/en/apps/pedometer, last accessed May, 7, 2014 
et al. 2003), it is not motivating most people to change their long-term behavior without further incentives (Swan 2013). The sole fact of using such a tracking system only influences shortterm behavior (Hawthorne effect, cf. Leonard \& Masatu 2006). In order to create long-lasting effects, the tracking platform providers offer incentives in the form of virtual badges or physical rewards as well as setting goals or sharing achievements on social platforms (Munson \& Consolvo 2012). However, even those extrinsic rewards fail for most users after some time (Walsh \& Golbeck 2014), as the desired behavior change (e.g. take more steps) is not triggered by a measure that can be well integrated in the people's daily lives.

For that reason, we propose a novel concept that includes the tracked daily fitness data in standard trip planning. By setting a daily physical activity goal, location preferences and special needs (such as barrier-free access) as well as standard trip parameters, the trip planning application calculates a route with walking segments so that the set physical activity goal (e.g., a certain amount of steps) can be reached.

\section{Concept and Prototype of Fitness-based Trip Planning}

Our concept primarily aims at users that need to increase their daily physical activity. This can, for example, be the case for certain medical indications, such as type 2 diabetes, obesity, or lack of exercise (Sigal et al. 2004). In that cases, patients often get prescribed a certain amount of steps per day, for example, 10,000 steps/day for healthy 'active' adults (Le-Masurier et al. 2003). The step count is normally tracked by standard mechanical or electronic pedometers.

Our concept allows embedding exercising in daily trips. This is enabled by coupling the current step count and the step history with a trip planning application. In order to be able to respond to the individual needs of the users, each user has to create an account and specify exercising goals as well as special needs. The goal can either be a specific step count that shall be reached on average per day, or a training program that gradually increases the exercise intensity to rise the initial daily step count average to the specified goal. Special needs define whether a user needs barrier-free access (e.g., no steps, max. inclination, even floor surfaces) or needs to transport something (e.g., walking aids or a stroller).

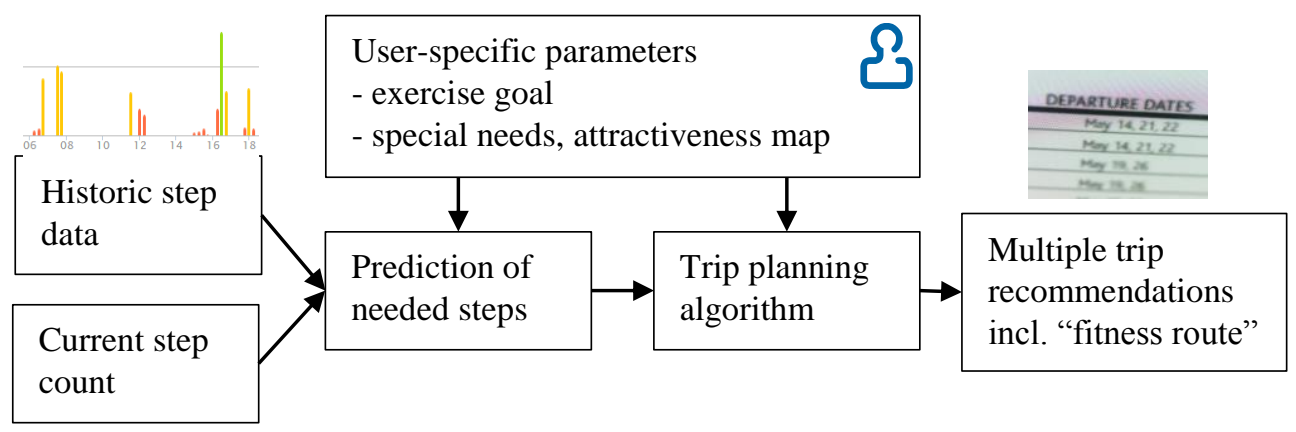

Figure 1: By comparing historic step data with the current number of steps, the step prediction algorithm determines the needed steps to reach the set goal. The trip planning algorithm 8.039 calculates appropriate trips including a "fitness route" with an extra walking segment. 

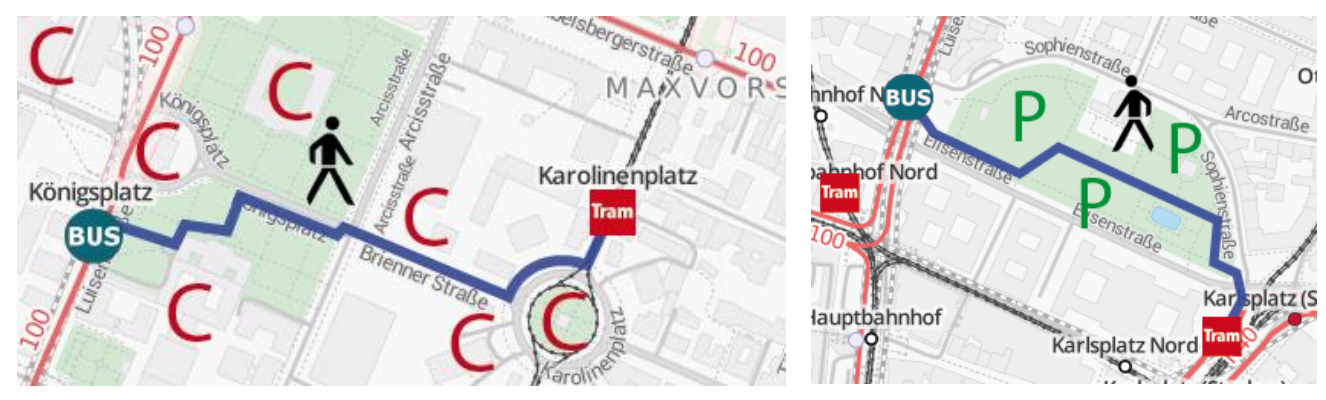

Figure 2: The algorithm uses a user-dependent attractiveness map to determine where the most enjoyable walking segment can be included in the "fitness route". The left image illustrates a walking segment in a cultural environment ( $C$ indicates cultural sites). In the right image, a walking segment in a park environment is depicted ( $P$ indicates park area). Walking segments are often inserted when transferring between different modes of transport. Map data: (C) OpenStreetMap contributors; Tram and bus logos: Munich Transport and Tariff Association

Users can further specify preferred training environments, such as cultural sites (e.g., historic, arts), parks, or shopping areas.

When a user wants to plan a new trip, the user opens the trip planning application and logs in into their personal account. The current step count of the user is fetched via the application programming interface (API) from a user specified cloud-based fitness tracking platform. By comparing the current step count with historical patterns, the trip planning platform calculates an estimation of steps that are needed to reach the specified goal on that day. Together with the defined needs, preferences, and entered trip parameters, the platform calculates several appropriate trip recommendations including a so-called "fitness route" with an additional walking segment so that the step count can be reached (cf. Fig. 1).

As users are more motivated when exercising is diversified but done in environments users are feeling well in (Munson \& Consolvo 2012), an attractiveness algorithm creates an attractiveness map for each user by comparing the environment nearby the standard routes with user preferences (see Fig. 2). The attractiveness map is adapted after each trip such that often visited places get lower attractiveness values and new walking segments are returned for next similar route recommendations. In future, the attractiveness values can also be adjusted by giving feedback after a trip or by including recommendations of similar users.

Our current prototype is based on the open source trip planning platform OpenTripPlanner (Hilsman \& Barbeau 2011) and uses OpenStreetMap data (Haklay \& Weber 2008) for calculating the routes. Schedules for public transportation are imported from GTFS (General Transit Feed Specification) files provided by the public transportation agencies. The attractiveness map is implemented statically and only distinguishes between cultural sites, parks and shopping areas. These maps are generated from the density of entries in the respective categories from OpenStreetMap data. The OpenTripPlanner platform performs a multi-factor optimization with user-dependent constrains (Dibbelt et al. 2012) to allow for barrier-free access when needed, and to minimize trip duration and number of transfers. The optimization also takes into account our contributed algorithms to maximize attractiveness and to reach the determined step count. The historic and current step count is retrieved from the hline-online.com platform that allows real-time data gathering from coupled pedometers. 


\section{Conclusion and Future Work}

We proposed a trip planning concept that includes physical activity data for helping users to reach defined fitness goals, and presented the setup of our first prototype. We argue that by embedding self-tracking information into everyday trip planning, users can more easily reach daily activity goals. We currently develop a mobile application that simplifies access to the trip planner (Diewald et al. 2012). As future work, we will add dynamic attractiveness maps and perform a comparison study with our implemented fitness trip planning platform.

\section{Acknowledgements}

This work was supported in part by the German Federal Ministry of Education and Research (BMBF, project PASSAge, funding number 16SV5748). Further information on the project PASSAge can be found on http://www.passage-projekt.de

\section{References}

Choe, E.K., Lee, N.B., Lee, B., Pratt, W., and Kientz, J.A. (2014). Understanding Quantified-Selfers' Practices in Collecting and Exploring Personal Data. Proc. CHI, ACM, pp. 1143-1152.

Dibbelt, J., Pajor, T., and Wagner, D. (2012). User-Constrained Multi-Modal Route Planning. Proc. ALENEX, Society for Industrial and Applied Mathemathics, pp. 118-129.

Diewald, S., Möller, A., Roalter, L., and Kranz, M. (2012). MobiliNet: A Social Network for Optimized Mobility. Adjunct Proc. AutomotiveUI, ACM, pp. 145-150.

Haklay, M., Weber, P. (2008). OpenStreetMap: User-Generated Street Maps. Pervasive Computing, 7(4), pp. 12-18.

Hillsman, E.L., and Barbeau, S.J. (2011). Enabling Cost-Effective Multimodal Trip Planners through Open Transit Data. Tampa, FL, USA:National Center for Transit Research.

Le-Masurier, G.C., Sidman, C.L., and Corbin, C.B. (2003). Accumulating 10,000 Steps: Does this Meet Current Physical Activity Guidelines? Research Quarterly for Exercise and Sport, 74(4), 389-394.

Leonard, K., and Masatu, M.C. (2006). Outpatient Process Quality Evaluation and the Hawthorne Effect. Social Science \& Medicine, 63(9), 2330-2340.

Möller, A., Roalter, L., Diewald, S., Scherr, J., Kranz, M., Hammerla, N. Olivier, P., and Plötz, T. (2012). GymSkill: A Personal Trainer for Physical Exercises. Proc. PerCom, IEEE, pp. 213-220.

Munson, S.A., and Consolvo, S. (2012). Exploring Goal-setting, Rewards, Self-monitoring, and Sharing to Motivate Physical Activity. Proc. PervasiveHealth, IEEE, pp. 25-32.

Sigal, R.J., Kenny, G.P., Wasserman, D.H., and Castaneda-Sceppa, C. (2004). Physical Activity/Exercise and Type 2 Diabets. Diabetes Care, 27(10), 2518-2539.

Swan, M. (2013). The Quantified Self: Fundamental Disruption in Big Data Science and Biological Discovery. Big Data. 1(2), 85-99.

Walsh, G., and Golbeck, J. (2014). StepCity: A Preliminary Investigation of a Personal Informatics-based Social Game on Behavior Change. CHI EA, ACM, pp. 2371-2376.

\section{Contact Information}

\{stefan.diewald, andreas.moeller, roalter\}@tum.de, matthias.kranz@uni-passau.de 